\title{
Minor Loops in the Harrison Model
}

\author{
K. ChWAStek ${ }^{a}$, J. SzCZYGeOWSkI ${ }^{a, b}$, W. WILCZYŃSKI ${ }^{b}$ \\ ${ }^{a}$ Faculty of Electrical Engineering, Al. Armii Krajowej 17, 42-200 Czȩstochowa, Poland \\ ${ }^{b}$ Institute of Electrical Engineering, ul. Pożaryskiego 28, 04-703 Warszawa, Poland
}

A novel description of hysteresis phenomenon is presented. The examined description emphasizes the roles of bistability and quantum phenomena as processes responsible for hysteresis loop formation in magnetic materials. Major and symmetric minor hysteresis loops are modelled in chosen soft magnetic materials using the Harrison model. A reasonable agreement between theory and experiment is achieved.

PACS: 75.60.-d, 75.60.Ej, 85.70.Ay

\section{Introduction}

The century-old concept of "effective field" introduced by Weiss [1] has withstood the test of time and remained one of the most inspiring ideas in contemporary ferromagnetism [2]. It can provide a useful framework for studying phase transitions and for thermodynamic considerations, what is of interest to solid-state physicists [311]. From the engineers' perspective the idea of effective field is attractive, as it allows to develop low-dimensional hysteresis models, which can be used e.g. for the description of magnetomechanical effects in nondestructive testing of material properties (NDT applications) [12-17].

Many description of hysteresis phenomenon may be expressed with the relationship [18]:

$$
M(t)=\hat{\Gamma}\left\{H_{\mathrm{eff}}(t)\right\},
$$

where $\hat{\Gamma}$ is the hysteresis operator and $H_{\text {eff }}$ is the effective field, given in the first approximation as $H_{\text {eff }}(t)=H(t)+\alpha M(t)$.

The notation of relationship (1) emphasizes the selfadjusting character of the relationship between the external field strength $H(t)$ and the internal magnetization $M(t)$. The role of positive feedback in the system as the most probable reason for hysteresis phenomenon, as implied from the relationship for $H_{\text {eff }}$, is pronounced in many papers $[3,4,6,7,12]$.

The study of bistability is not only interesting from the scientific point of view, but it might be useful in engineering applications. Those include e.g. nonlinear optics [19], chaos control [20, 21] or sophisticated microand nanodevices used as sensors [22-25].

Recently a novel description of hysteresis phenomenon has been advanced by Harrison [26-28]. Despite its simplicity the model provides some useful insights into the physics of magnetization process. The aim of the present paper is to verify the ability of Harrison model to describe minor loops of chosen soft magnetic materials, commonly used in electrical engineering.

\section{Model description}

\subsection{Model equations in normalized form}

The original description [26] describes a head-to-tail alignment of magnetic moments within the ferromagnetic material with the quantum-mechanical Brillouin function for $J=1 / 2$, written in the normalized form as

$$
y=\tanh \left(\frac{x+y}{\tau}\right)=\tanh \left(\frac{x_{\mathrm{e}}}{\tau}\right),
$$

where $y$ is interpreted as reduced magnetization, the meaning of $x$ is reduced field strength, whereas $\tau$ is temperature referred to Curie point. The relationship (2) describes a bistable rectangular loop. It can be inverted to yield the $x_{\text {hyst }}(y)$ relationship

$$
x_{\text {hyst }}=\tau \operatorname{arctanh}(y)-y \text {. }
$$

A more realistic sigmoid shape of hysteresis loop can be obtained by consideration of another term of field strength, related to reversible magnetization process. In the proposed model the reversible magnetization process is described with the unmodified Langevin function

$$
y=\mathcal{L}\left(x_{\mathrm{anh}}\right)=\operatorname{coth} \frac{x_{\mathrm{anh}}}{\gamma}-\frac{\gamma}{x_{\mathrm{anh}}},
$$

where $\gamma$ is a parameter. It is assumed that the positivefeedback field strength responsible for hysteresis and the anhysteretic field strength can be added together to obtain a complete description of hysteresis phenomenon, yielding

$$
x=x_{\text {hyst }}(y)+x_{\text {anh }}(y) .
$$

The main drawback of the original model is its ability to describe major hysteresis loops only.

In the subsequent paper [27] the model has been extended to take into account the initial magnetization curve by the introduction of a functional dependence $f_{\beta}$, which can be given as inverse Gaussian

$$
f_{\beta}(x+y)=1-d \exp \left\{-\frac{1}{2}\left[\frac{(x+y)}{\sigma}\right]^{2}\right\} .
$$

The modified relationship (2) has become

$$
y_{\text {hyst }}=\tanh \left[\frac{(x+y) f_{\beta}(x+y)}{\theta}\right] \text {, }
$$

where $\theta$ is the normalized temperature, different from $\tau$ in the original description [26]. For a detailed discussion the readers are referred to [27]. It seems important to stress that the presented model is able to describe and interpret different types of initial magnetization curves (either nucleation or pinning dominated ones, cf. [2, p. 318], as well as shapes of hysteresis loops different from the most commonly encountered sigmoid one, like wasp-waisted loops. Moreover the author claims his description is valid for magnetic materials with intrinsic coercive field strengths 
ranging over six orders of magnitude.

An important step forward has been achieved in [28], where a theoretical approach to model first order reversal curves has been proposed. The author has suggested that a const- $f_{\beta}$ description might be valid for the whole $y-x$ plane apart from the initial magnetization curve, which requires a separate $f_{\beta}$ functional dependence. The consequence of this assumption is the possibility to recover the shapes of minor hysteresis loops and reversal curves from the shape of the major i.e. saturating loop. A similar concept has been expressed in a number of papers on hysteresis modelling [29-36].

\subsection{Model equations in physical units}

It is advantageous to express the equations of Harrison model in physical units in order to recognize the admissible ranges of model parameters and to obtain realistic modelled hysteresis loops.

The most important idea in the description is the linear addition of field strength contributions related to distinct physical phenomena occurring in different time- and space scales, cf. Eq. (5). The concept that field strength summation might be carried out within the framework of effective field has also been expressed elsewhere [37]. Equation (2), which describes the irreversible rectangular loop, in physical units becomes

$$
M=M_{\mathrm{s}} \tanh \left[\frac{\mu_{0} m_{\mathrm{B}}}{k_{\mathrm{B}} T} \beta\left(H_{\text {hyst }}+\alpha M\right)\right],
$$

where $\mu_{0}$ is free space permeability, $\mu_{0}=4 \pi \times 10^{-7}$ $[\mathrm{H} / \mathrm{m}], m_{\mathrm{B}}$ is Bohr magneton, $m_{B}=9.274 \times 10^{-24}\left[\mathrm{Am}^{2}\right]$, $k_{\mathrm{B}}$ is Boltzmann's constant, $k_{\mathrm{B}}=1.381 \times 10^{-23}[\mathrm{~J} / \mathrm{K}]$, whereas $T[\mathrm{~K}]$ is the absolute temperature (assumed to be equal to $300 \mathrm{~K}$ during the measurements). $\alpha$ is the Weiss' dimensionless mean field parameter, whose value is approximately equal to $H_{\mathrm{c}} / M_{\mathrm{s}}$ [38]. $\beta$ is the new dimensionless parameter introduced by Harrison in order to describe the cooperative action of Bohr magnetons within a magnetic domain. $M_{\mathrm{s}}$ expressed in $[\mathrm{A} / \mathrm{m}]$ is the technical saturation magnetization at temperature $T$.

Equation (4), which describes the reversible component of magnetization, is given in physical units as

$$
M=M_{\mathrm{s}}\left[\operatorname{coth}\left(H_{\mathrm{anh}} / \gamma\right)-\gamma / H_{\mathrm{anh}}\right] .
$$

It can be easily noticed that $\gamma$ is expressed in the units of field strength, $[\mathrm{A} / \mathrm{m}]$.

In order to determine the inverse Langevin function we have used the approximation advanced by Cohen [39] and recalled recently by Arrott [40]

$$
H_{\mathrm{anh}}=\gamma m \frac{3-m^{2}}{1-m^{2}},
$$

where $m=M / M_{\mathrm{s}}$.

\subsection{Comparison with other hysteresis models}

The description proposed by Harrison bears some similarities with other hysteresis models, in particular the $T(x)$ model advanced by Takács [41] and the JilesAtherton proposal [12]. In the Takács model the reversible magnetization component is linear, whereas the non-linear, irreversible magnetization component is described with the hyperbolic tangent function. A decomposition of hysteresis loop into the reversible (related to domain wall rotation) and the irreversible parts (related to domain wall translation through pinning sites) has been demonstrated by Varga et al. for the case of triangular $H$-excitation [42]. The idea that the irreversible and reversible magnetization processes overlap in the whole $M-H$ plane, but can be decoupled and separately examined is common for the Takács and the Harrison models.

A much more complex situation is with the JilesAtherton (JA) description, where both magnetization components are coupled through the effective field term, but moreover through a magnetization dependent $R(m)$ function in a recent model modification [36, 43, 44]. Anhysteretic magnetization is described in the JA formalism with the modified Langevin function with respect to the effective field. On the other hand, the anhysteretic magnetization in the Harrison description is a function of external field strength only. The descriptions advanced by Jiles and Harrison differ significantly as far as the role of pinning sites within the ferromagnetic material is considered, as already raised by the second author himself [26, 28]. Jiles and Atherton assume the existence of pinning sites as the cause for hysteresis phenomenon. In their description the hysteresis loop branches are obtained by offsetting the irreversible magnetization from the anhysteretic magnetization. A quantity proportional to the product of density of pinning sites and their energy is a weighting parameter in the JA ordinary differential equation, which may be easily solved either for $H$ - or $B$-excitation case.

Total magnetization $M$ plays the role of state variable in Jiles-Atherton and Harrison descriptions alike.

\section{Modelling}

The measurements of major and symmetric minor hysteresis loops were carried out for two chosen soft magnetic materials, used commonly in electrical engineering - an amorphous Co-based alloy AMMET 212 in the form of toroid core and a non-oriented alternator steel V350-50A in the form of Epstein strips. The measurement setup fulfilled all the requirements of the appropriate IEC 60404 standards. Sine flux wave was imposed into the system ( $B$-excitation case), the form factor of the output voltage was approximately equal to 1.11. Further details concerning the measurement setup may be obtained from the website [45]. The excitation frequency was kept as low as possible in order to avoid the disturbing effect of eddy currents on the shape of measured hysteresis loops.

In the Harrison description the modelled hysteresis loop consists of upper and lower segments of the $S$-shaped curve obtained from the solution of equation

$$
\begin{gathered}
H_{\text {model }}=\frac{k_{\mathrm{B}} T}{\mu_{0} m_{\mathrm{B}} \beta} \operatorname{arctanh} \frac{M}{M_{\mathrm{s}}} \\
-\alpha M+\gamma m \frac{3-m^{2}}{1-m^{2}},
\end{gathered}
$$


and sigmoid reversible curves obtained by offsetting horizontally the anhysteretic Langevin function by the value $\pm H_{\mathrm{c}}$. The intermediate part of the $S$-shaped curve is not observable, yet it is possible that it manifests itself in the so-called re-entrant hysteresis loops, obtained in measurements e.g. on single, high-purity $3.2 \%$ SiFe crystals, cf. [2, p. 322]. The model equations may be recast into the normalized form by referring the $M$ and $H$ values to $M_{\mathrm{s}}$ and $\alpha M_{\mathrm{s}}$, respectively. Then it is sufficient to know the coercive field strength and the amplitude of a symmetric minor loop to recover its shape using a reverse transformation from the normalized into the physical units.

Table comprises the estimation results for the considered soft magnetic materials.

TABLE

Estimation results for the considered materials

\begin{tabular}{c|c|c}
\hline \hline & AMMET 212 & V350-50A \\
\hline$\alpha[-]$ & $9.69 \times 10^{-6}$ & $7.214 \times 10^{-5}$ \\
$\beta[-]$ & $2.23 \times 10^{8}$ & $9.98 \times 10^{8}$ \\
$\gamma[\mathrm{A} / \mathrm{m}]$ & 0.44 & 37.51 \\
$M_{s}[\mathrm{~A} / \mathrm{m}]$ & $8.12 \times 10^{5}$ & $1.206 \times 10^{6}$
\end{tabular}

For the estimation of model parameters the MATLAB implementation of the robust DIRECT algorithm [46] is used. The algorithm implements the Banach's contraction theorem in $n$-dimensions simultaneously. Its important advantage is that the dimensions of the search space are normalized into unit segments, thus the solution sensitivity against variations in any direction is indirectly controlled. The toolbox has already been successfully applied for estimation of Jiles-Atherton model parameters [38].

Figure 1 depicts the measured and the modelled major loop for AMMET 212, $B_{\mathrm{m}}=1$ [T], whereas Fig. 2 presents the measured and modelled minor loop for this material at $B_{\mathrm{m}}=0.5[\mathrm{~T}]$. In Fig. 3 some modelling results concerning minor loops in the non-oriented alternator steel, grade V350-50A are presented.

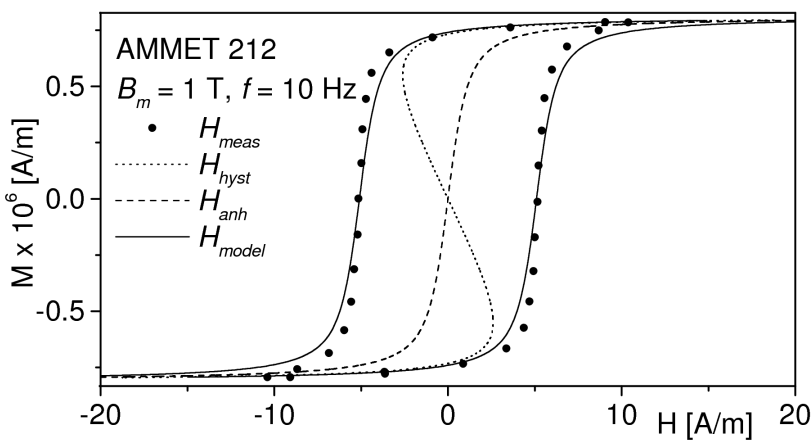

Fig. 1. The measured and the modelled major loops for AMMET 212.

On the basis of the presented results it can be stated that a reasonable agreement between theory and experiment has been achieved. The validity of Harrison model

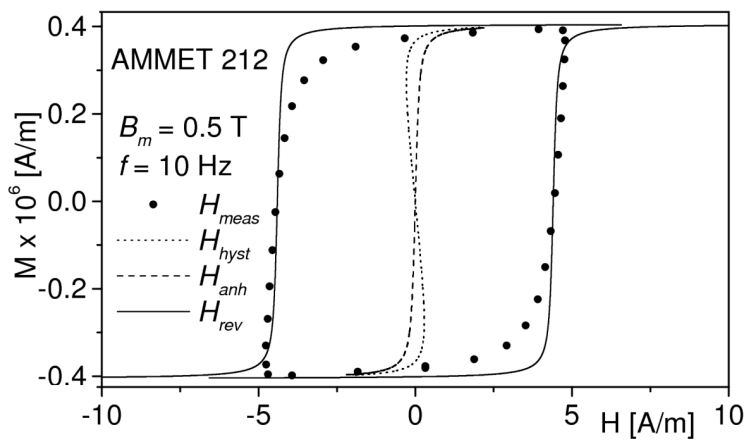

Fig. 2. The measured and the modelled minor loops for AMMET 212.

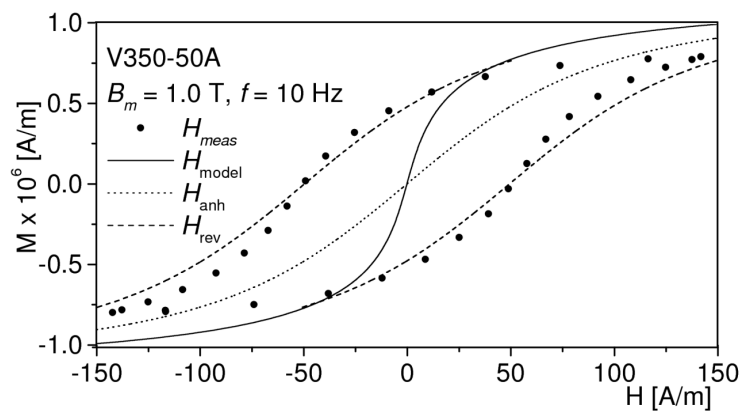

Fig. 3. The measured and the modelled minor loops for V350-50A alternator steel.

should however be further supported with the results of modelling and experiments carried out in a wide temperature range including Curie point $[5,7,9]$, what is beyond our laboratory capabilities.

It can be also concluded that the use of Brillouin function or its specific cases (hyperbolic tangent or Langevin function) for modelling hysteresis in soft magnetic materials is adequate, as already pointed out in a number of papers $[5,12,35,41,43,44]$.

The description of anhysteretic processes has been the subject of intensive studies due to their role in the magnetomechanical effects and thermodynamics [14, 47]. Recently Takács has noticed, that the knowledge of either the hysteretic or the anhysteretic curve makes it possible to describe the behaviour of magnetic material, as they both carry an equivalent piece of information on remagnetization history [48]. Some years ago Atherton et al. have conjectured, that in fact there might exist families of offset anhysteretic curves [13], but no explanation for this phenomenon has been provided. Sablik and Langman have envisaged anhysteretics as surfaces in the $M-H-\sigma$ space, where $\sigma$ denoted the external stress [49]. By analogy one may consider anhysteretic surfaces in the $M-H-T$ space. For a constant ambient temperature one obtains different offset anhysteretic curves, which correspond to different reversal curves, defined by magnetization values at the beginning and at the end of the magnetization process. This concept has been imple- 
mented for the modified JA description in [36], where two JA model parameters have been updated according to some power laws with respect to relative magnetization level. In the Harrison model the update of anhysteretic curve (and, what is more noticeable - of the $S$-shaped hysteretic curve) for the minor loops is achieved by the normalization of model equations. This effect is clearly visible in Fig. 1 and 2. The existence of similar relationships, useful for monitoring material aging in NDT, has been proven experimentally in a number of recent papers, cf. e.g. $[34,50]$.

\section{Conclusions}

A novel description has been applied for modelling major and symmetric minor hysteresis loops in chosen soft magnetic materials. A reasonable agreement between theory and experiment has been achieved.

\section{Acknowledgements}

The work has been supported by a research grant N N510 702540 from National Centre of Science.

\section{References}

[1] P. Weiss, J. Phys. Theor. Appl. 6, 661, (1907).

[2] G. Bertotti, Hysteresis in magnetism San Diego:Academic Press, (1998).

[3] A. Visintin, Physica B 275, 87, (2000).

[4] Št. Barta, P. Dieška, J. Electr. Eng. 51, 113, (2000).

[5] Gy. Kádár, Zs. Szabó, J. Magn. Magn. Mater. 272276, e547, (2004).

[6] F. Šrobár, Physica B 372, 21, (2006).

[7] M. Fabrizio, C. Giorgi, A. Morro, Int. J. Eng. Sci. 47, 821, (2009).

[8] D. Vollath, F.D. Fischer, J. Nanopart. Res. 11, 1485, (2009).

[9] A. Raghunathan, Y. Melikhov, J.E. Snyder, D.C. Jiles, IEEE Trans. Magn. 46, 1507, (2010).

[10] D. Vollath, F.D. Fischer, Prog. Mater. Sci. 56, 1030, (2011).

[11] V.I. Zverev, R.R. Gimaev, A.M. Tishin, Ya. Mudryk, K.A. Gschneider, V.K. Pecharsky, J. Magn. Magn. Mater. 323, 2453, (2011).

[12] D.C. Jiles, D.L. Atherton, J. Magn. Magn. Mater. 61, 48, (1986).

[13] D.L. Atherton, V. Ton, IEEE Trans. Magn. 26, 1153 , (1990).

[14] D.C. Jiles, J. Phys. D: Appl. Phys. 28, 1537, (1995).

[15] H.W.L. Naus, IEEE Trans. Magn. 38 3417, (2002).

[16] O. Perevertov, J. Phys. D: Appl. Phys. 36, 785, (2003).

[17] R. Szewczyk, J. Phys. D: Appl. Phys. 40, 4109, (2007).

[18] P. Andrei, A. Stancu, H. Hauser, P. Fulmek, J. Optoelectr. Adv. Mater. 9, 1137, (2007).

[19] P. Jung, G. Gray, R. Roy, P. Mandel, Phys. Rev. Lett. 65, 1873, (1990)

[20] L. Yaowen, P. Huican, Z. Hong, W. Yinghai, Phys. Lett. A 262, 416, (1999 ).
[21] S. Lynch, Nonlin. Anal. Th. Meth. Appl. 47, 4501, (2001).

[22] K.R. Pirota, M. Provencio, K.L. Garcia, R. EscobarGalindo, P. Mendoza Zelis, M. Hernández-Vélez, M. Vázquez J. Magn. Magn. Mater. 290-291, 68, (2005).

[23] P.D. Dimitropoulos, G.I. Stamoulis, E. Hristoforou IEEE Sens. J. 6, 721, (2006).

[24] H. Chiriac, T.A. Óvári, P. Pascariu J. Appl. Phys. 103, 07D919, (2008).

[25] I. Petrila, A. Stancu Physica B 406, 906, (2011).

[26] R.G. Harrison, IEEE Trans. Magn. 39, 950, (2003).

[27] R.G. Harrison, IEEE Trans. Magn. 40, 1506, (2004).

[28] R.G. Harrison, IEEE Trans. Magn. 45, 1922, (2009).

[29] D.C. Jiles IEEE Trans. Magn. 28, 2602, (1992).

[30] S.E. Zirka, Yu.I. Moroz, IEEE Trans. Magn. 31, 3509, (1995).

[31] M. Esguerra, J. Magn. Magn. Mater. 157/158, 366, (1996).

[32] D. Lederer, H. Igarashi, A. Kost, T. Honma, IEEE Trans. Magn. 35, 1211, (1999).

[33] S.E. Zirka, Yu.I. Moroz, IEEE Trans. Magn. 35 , 2090, (1999).

[34] S. Takahashi, S. Kobayashi, Y. Kamada, H. Kikuchi, L. Zhang, K. Ara, Physica B 372, 190, (2006).

[35] Z. Włodarski, J. Magn. Magn. Mater. 308, 15, (2007).

[36] K. Chwastek, J. Phys. D: Appl. Phys. 42, 1652002, (2009).

[37] C.S. Schneider, S.D. Winchell, Physica B 372, 269 (2006).

[38] K. Chwastek, J. Szczygłowski, J. Magn. Magn. Mater. 314, 47, (2007).

[39] A. Cohen, Rheol. Acta 30, 270, (1991).

[40] A.S. Arrott, J. Appl. Phys. 103, 07C715, (2008).

[41] J. Takács, Mathematics of hysteretic phenomena, Weinheim:Wiley-VCH, (2003).

[42] L.K. Varga, Gy. Kovács, J. Takács, J. Magn. Magn. Mater. 320, L26, (2008).

[43] K. Chwastek , J. Phys. D: Appl. Phys. 43, 015005, (2010).

[44] K. Chwastek, J. Szczygłowski, Arch. Electr. Eng. 60, 49, (2011)

[45] J. Bajorek, http://www.rjmeasurement.com.pl.

[46] D.E. Finkel, Global optimization with the DIRECT algorithm, PhD Thesis, North Carolina State University, Raleigh, (2005).

[47] J. Pearson, P.T. Squire, D. Atkinson, IEEE Trans. Magn. 33, 3970, (1997).

[48] J. Takács, Physica B 372, 57, (2006).

[49] M. Sablik, R.A. Langman, J. Appl. Phys. 79, 6134, (1996).

[50] S. Takahashi, S. Kobayashi, J. Appl. Phys. 107, 063903, (2010). 